\title{
基于特征点的空间目标三维位姿单目视觉确定 算法
}

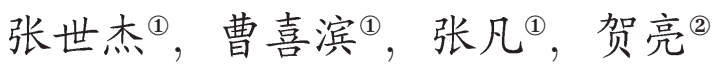

(1) 哈尔滨工业大学卫星技术研究所，哈尔滨 150080

(2) 上海航天控制工程研究所研发中心, 上海 200233

* 通信作者. E-mail: sjzhang@hit.edu.cn

收稿日期: 2009-01-14; 接受日期: 2009-05-12

国家安全重大基础研究项目 (批准号: 51312)、教育部长江学者和创新团队发展计划 (批准号: IRT0520) 和高等学校博士学科点专项 科研基金 (批准号: 20070213055) 资助项目

摘要 针对基于特征点的空间目标包括相对位置和相对姿态等的三维位姿单目 视觉确定问题, 提出了一种基于逆投影思想的迭代方法. 给出了一种包含景深估 计和绝对方位解算两阶段的迭代算法, 在景深估计阶段首先计算由转移矩阵表示 的最优平移矢量, 然后重构各特征点, 并利用其在逆投影线上投影更新各特征点的 景深; 在绝对方位解算阶段采用 Umeyama 绝对方位解析算法计算相对姿态矩阵, 上述两阶段迭代进行直至结果收敛。利用全局性收敛性定理证明了文中算法的全 局收玫性. 最后, 以航天器交会对接最终逼近段的视觉测量为背景对该算法进行 了数学和物理仿真, 进一步验证了算法的有效性和收玫性.

关键词

相对位姿

单目视觉

特征点

绝对方位

景深估计

\section{1 引言}

三维位姿确定是指求解两个坐标系间的相对位姿关系, 包括 3 个平移量和分别绕 3 个坐标轴的 旋转量. 基于点特征的单目位姿确定方法通过单个相机对目标成像, 利用目标像中 3 个以上非共线特 征点的像坐标求解目标与相机间的相对位置和相对姿态, 由于其结构简单、易于实现等特点成为视觉 测量领域研究热点之一, 并广泛应用于汽车、机器人、飞机、航天器等领域的非接触式测量任务 ${ }^{[1]}$.

在相机内参数已知的情况下, 由特征点与其像点的对应关系求解目标相对于相机的位姿就是经典 的 $\mathrm{P} n \mathrm{P}$ 问题, 从上世纪 80 年代以来已有众多国内外学者给出了大量的 $\mathrm{P} n \mathrm{P}$ 问题解法 ${ }^{[2]}$, 这些算法大 致可分为迭代算法和非迭代算法两类. 非迭代算法采用代数方法直接求出相对位姿, 针对 P3P、P4P 及 P5P 等问题, 推导出多种解析算法 ${ }^{[3 \sim 5]}$, 该类算法具有运算量小、计算速度快等优点, 但受误差影 响大且精度不高, 可应用于迭代算法的初值计算. 对于 6 个以上异面特征点或有 4 个以上共面特征点 的情况, 可以进行线性求解, 因此另一类非迭代算法是将 $\mathrm{P} n \mathrm{P}$ 问题求解转化代数距离最小化问题的线 性方法 ${ }^{[6 \sim 10]}$, 进一步可通过奇异值分解等方法加以求解, 但该类方法的计算量会随特征点数的增加而 急剧增大. Lepetit 等 ${ }^{[11]}$ 提出了一种计算量为 $O(n)$ 的 $\mathrm{P} n \mathrm{P}$ 问题非迭代算法, 其基本思路是将所有特 
征点表示为 4 个虚拟控制点, 从而将 $\mathrm{P} n \mathrm{P}$ 问题转化为估计 4 个虚拟控制点在相机坐标系的坐标值问 题, 但该方法的精度同迭代算法相比仍然较低.

由于基于不存在像噪声假设下推导出的准确解对于像点位置误差极为敏感, 因此为克服噪声影响, 提高位姿确定精度, 一般采用迭代算法求解 $\mathrm{P} n \mathrm{P}$ 问题, 一种处理方式是将 $\mathrm{P} n \mathrm{P}$ 问题表示为受约束的 非线性优化问题, 通过求解该优化问题得到目标相对位姿的数值解 ${ }^{[12]}$. 然而, 该类方法是一个优化变 量空间为 $N+6$ 维 ( $N$ 为点特征数) 的非线性优化问题, 计算量较大, 且受初始值的影响算法通常会收 敛到局部最小值而不是全局最小值, 或收敛到错误解.

针对基于优化方法的迭代算法所面临的问题, 部分学者提出了一类不断修正运动参数的迭代算法, 使得根据运动参数所计算出的像点位置和实际观察到的像点位置尽可能接近. Dementhon 等 ${ }^{[13]}$ 提出 了一种基于弱透视投影相机模型的相对位姿确定迭代算法. Haralick 等 [14] 提出了一种基于点特征同 时计算日标位姿和特征点景深的位姿确定算法, 该算法通过引入特征点景深变量消除了由于透视投影 所产生位姿确定问题的非线性, 且具有全局收玫性, 然而该算法局部收玫速度较慢. $\mathrm{Lu}$ 等 ${ }^{[15]}$ 通过定 义物空间共线误差作为目标函数, 将位姿确定问题转化为物空间共线误差最小化问题, 采用顺序求解 旋转矩阵和平移矢量的迭代方法, 提出了一种具有全局收玫性的正交迭代 (orthogonal iteration) 算法, 该算法具有迭代次数少、计算速度快等优点, 适用于工业领域应用的实时性要求. 在文献 [15] 的基础 上, Schweighofer 等 $[16,17]$ 分别提出了针对平面目标的鲁棒位姿估计算法, 以及针对通用相机模型的具 有全局收敛的 $\mathrm{P} n \mathrm{P}$ 问题迭代算法.

受 Haralick 和 $\mathrm{Lu}$ 等研究的启发, 本文提出了一种基于逆投影思想的包括景深计算和绝对定向求 解两阶段的快速全局收玫迭代算法, 并理论证明该算法的全局收玫性. 最后以航天器交会对接单目视 觉测量任务为应用背景, 对本文算法进行数学仿真和物理仿真验证, 以验证算法的可行性和有效性.

\section{2 问题描述 (problem formulation)}

\section{1 坐标定义}

分别定义相机参考坐标系、像平面坐标系和目标参考坐标系, 其中相机坐标系原点在投影中心 $O$, $Z$ 轴与投影轴重合并指向目标, $X$ 轴、 $Y$ 轴与像平面的坐标轴 $U$ 和 $V$ 平行, $X Y$ 平面与像平面的距 离为相机焦距 $f$.

\section{2 相机模型}

目标特征点在目标坐标系中的坐标为 $\left\{\boldsymbol{s}_{i}:\left(x_{i}, y_{i}, z_{i}\right), i=1,2, \ldots, N\right\}$, 其在相机坐标系中的坐标 为 $\left\{\boldsymbol{S}_{i}^{\prime}:\left(X_{i}^{\prime}, Y_{i}^{\prime}, Z_{i}^{\prime}\right)\right\}$, 像点坐标为 $\left\{\boldsymbol{S}_{i}:\left(X_{i}, Y_{i}\right)\right\}$, 由刚体运动的一般方程可知:

$$
\boldsymbol{S}_{i}^{\prime}=\boldsymbol{M} \boldsymbol{s}_{i}+\boldsymbol{t}, \quad i=1,2, \ldots, N
$$

其中 $\boldsymbol{t}=\left(t_{x}, t_{y}, t_{z}\right)^{\mathrm{T}}$ 和 $\boldsymbol{M}$ 分别为目标相对于相机坐标系的平移矢量和旋转矩阵. 目标特征点 $\boldsymbol{S}_{i}^{\prime}$ : $\left(X_{i}^{\prime}, Y_{i}^{\prime}, Z_{i}^{\prime}\right)$ 与像点坐标 $\boldsymbol{S}_{i}:\left(X_{i}, Y_{i}\right)$ 的关系由小孔成像相机模型表示,

$$
X_{i}=f \frac{X_{i}^{\prime}}{Z_{i}^{\prime}}, Y_{i}=f \frac{Y_{i}^{\prime}}{Z_{i}^{\prime}}, \quad i=1,2, \ldots, N .
$$

(1) 和 (2) 式为基于小孔成像模型的 $3 \mathrm{D}$ 空间坐标系到 $2 \mathrm{D}$ 图像坐标系的刚体转换一般方程组. 


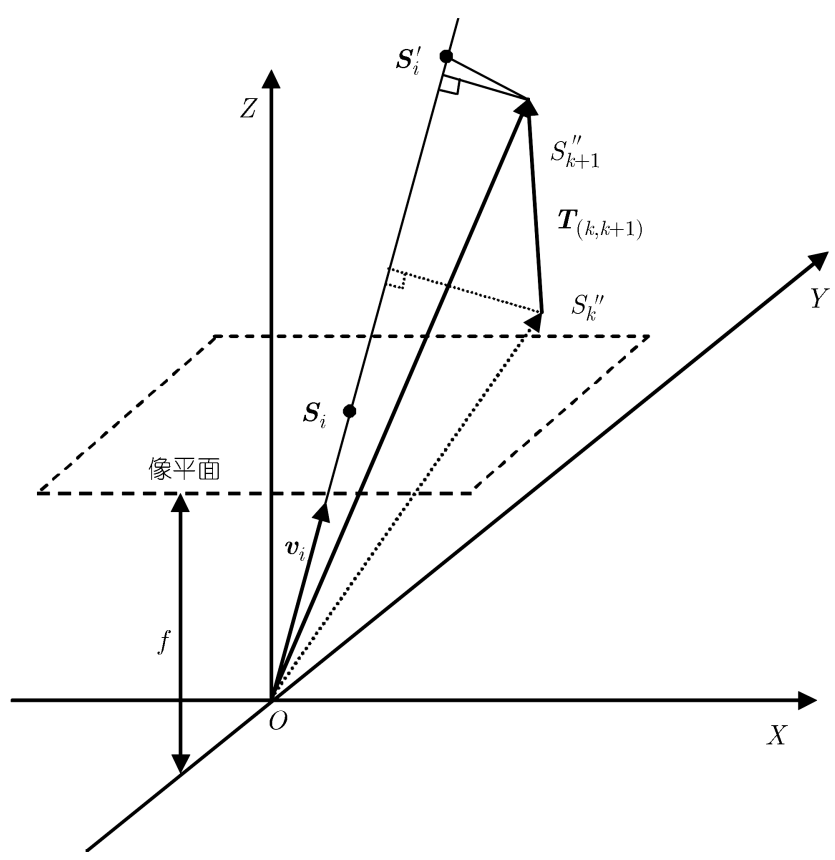

图 1 物空间内物像共线误差示意图

\section{3 共线方程}

所有目标特征像点都对应一条从相机投影中心出发的射线, 该射线穿过特征像点指向目标, 由于 该射线方向与目标特征点的投影线相反, 称为逆投影线, 如图 1 所示, 其单位矢量 $\boldsymbol{v}_{i}$ 可表示为

$$
\boldsymbol{v}_{i}=\frac{1}{\sqrt{\left(X_{i}^{2}+Y_{i}^{2}+f^{2}\right)}}\left(\begin{array}{c}
X_{i} \\
Y_{i} \\
f
\end{array}\right), \quad i=1,2, \ldots, N .
$$

理想情况下像点 $\boldsymbol{S}_{i}$ 和物点 $\boldsymbol{S}_{i}^{\prime}$ 在逆投影线 $\boldsymbol{v}_{i}$ 上, 称之为共线条件, 因此特征点 $\boldsymbol{S}_{i}^{\prime}$ 在相机坐标系 中的坐标可表示为

$$
\boldsymbol{S}_{i}^{\prime}=d_{i} \boldsymbol{v}_{i}, \quad i=1,2, \ldots, N,
$$

其中 $d_{i}$ 为目标特征点 $\boldsymbol{S}_{i}^{\prime}$ 距离投影中心的景深. 共线条件也可表述为: 特征点 $\boldsymbol{S}_{i}^{\prime}$ 在逆投影线 $\boldsymbol{v}_{i}$ 上的 投影等于其白身, 因此共线方程也可表示为

$$
\boldsymbol{S}_{i}^{\prime}=\boldsymbol{v}_{i}^{\mathrm{T}} \boldsymbol{S}_{i}^{\prime} \boldsymbol{v}_{i}=\boldsymbol{v}_{i}^{\mathrm{T}}\left(\boldsymbol{M} \boldsymbol{s}_{i}+\boldsymbol{t}\right) \boldsymbol{v}_{i}, \quad i=1,2, \ldots, N
$$

\subsection{Haralick 迭代算法}

由于图像处理过程中存在误差, 目标特征点通常不在由特征像点所重构的逆投影线上, 由 (4) 式 表示的 $\boldsymbol{S}_{i}^{\prime}$ 坐标与 (1) 式表示的实际坐标有一定误差, 可以利用该误差定义日标函数:

$$
e^{2}\left(\boldsymbol{M}, \boldsymbol{t},\left\{d_{i}\right\}\right)=\sum_{i=1}^{N}\left\|d_{i} \boldsymbol{v}_{i}-\left(\boldsymbol{M} \boldsymbol{s}_{i}+\boldsymbol{t}\right)\right\|^{2}, \quad \boldsymbol{M} \boldsymbol{M}^{\mathrm{T}}=\boldsymbol{I} .
$$


基于单目视觉的相对位姿确定问题可描述为: 给定目标特征点在目标坐标系中的物点坐标 $s_{i}$ : $\left(x_{i}, y_{i}, z_{i}\right), i=1,2, \ldots, N$, 及其相应的像点坐标 $\left\{\boldsymbol{S}_{i}:\left(X_{i}, Y_{i}\right), i=1,2, \ldots, N\right\}$, 求解旋转矩阵 $\boldsymbol{M}$ 、 平移矢量 $\boldsymbol{t}$ 和景深 $\left\{d_{i}, i=1,2, \ldots, N\right\}$, 使得 (6) 式表示的目标函数取最小值. 从而将相对位姿确定转 化为非线性优化问题, 可采用非线性最小二乘等算法求解, 但该优化问题是一个优化变量空间为 $N+6$ 维的非线性问题, 随着参与计算的特征点数目增多, 其计算量将急剧增加, 无法满足实际应用中实时 运算要求.

为克服传统非线性优化算法求解 (6) 式给出的优化问题面临的计算量大、收玫性差等缺点, Haralick 等提出了一种同时计算目标位置和目标特征点景深的确定算法 ${ }^{[14]}$, 该算法将相对位姿确定问题 分解为景深计算和绝对方位解算两个阶段, 上述两个阶段迭代进行直至结果收玫. 由于在该算法中引 入了景深变量, 消除了小孔成像透视投影所导致的非线性问题. 在 Haralick 迭代算法的绝对方位求解 阶段采用了基于旋转矩阵特征值分解的 Umeyama 解析算法, 在景深计算阶段, 采用了如下景深更新 算法:

$$
d_{i}^{(k+1)}=\boldsymbol{v}_{i}^{\mathrm{T}}\left(\boldsymbol{M}^{(k)} \boldsymbol{s}_{i}+\boldsymbol{t}^{(k)}\right) .
$$

由于 Haralick 迭代算法的两阶段都是解析求解, 避免了繁琐的非线性优化计算过程, 极大地降低 了计算复杂度. 然而, 数学仿真表明该算法的收玫速度过慢 (需要数百次迭代), 特别是在大的初始偏 差情况下可能导致算法结果错误. 参考图 1, 从几何意义上来讲 (7) 式成立的假设为: 第 $k+1$ 次的景 深值近似为特征点物空间坐标的第 $k$ 次迭代值在逆投影线上的投影长度. 实际上只有当特征点物空间 坐标的第 $k$ 次和第 $k+1$ 次迭代充分接近时上述假设才成立, 而在初始偏差较大的情况下该假设不再 成立; 另外 (7) 式给出的第 $k+1$ 次景深更新仍使用第 $k$ 次相对位姿 $\boldsymbol{M}^{(k)}, \boldsymbol{t}^{(k)}$, 这是 Haralick 迭代算 法收敛速度过慢甚至可能导致算法结果错误的主要原因.

\section{3 相对位姿确定的两阶段迭代算法}

\section{1 两阶段迭代算法}

虽然 Haralick 算法具有全局收玫性, 但其过慢的收玫速度限制了其应用, 针对这一问题, 本文在 Haralick 算法的基础上, 提出了一种新的基于逆投影思想的空间目标三维位姿单目视觉确定的迭代算 法, 该算法分为景深估计和绝对方位解算两阶段, 在景深估计阶段首先在相对旋转矩阵已知的前提下 计算最优平移矢量, 并计算各特征点的景深 $\left\{d_{i}, i=1,2, \ldots, N\right\}$, 在绝对方位解算阶段利用特征点 3 维 重构坐标 $\boldsymbol{S}_{i}^{\prime}$ 和 3 维模型坐标 $\boldsymbol{s}_{i}$, 并基于 $3 \mathrm{D}-3 \mathrm{D}$ 绝对方位解算解析算法计算出空间目标的相对旋转 矩阵, 上述两阶段迭代进行直至结果收玫.

\subsection{1 景深估计}

将 (4) 和 (5) 式代入 (6) 式可得

$$
e^{2}(\boldsymbol{M}, \boldsymbol{t})=\sum_{i=1}^{N}\left\|\left(\boldsymbol{M} \boldsymbol{s}_{i}+\boldsymbol{t}\right)-\boldsymbol{v}_{i}^{\mathrm{T}}\left(\boldsymbol{M} \boldsymbol{s}_{i}+\boldsymbol{t}\right) \boldsymbol{v}_{i}\right\|^{2},
$$

其中旋转矩阵 $M$ 满足约束 $\boldsymbol{M} \boldsymbol{M}^{\mathrm{T}}=\boldsymbol{I}$.

(8) 式是平移矢量 $\boldsymbol{t}$ 的二次型函数, 在给定旋转矩阵 $\boldsymbol{M}$ 的情况下, 能够求解出最优平移矢量 $\boldsymbol{t}$. 
对 (8) 式相对 $\boldsymbol{t}$ 求偏导并令其为零得

$$
\frac{\partial e^{2}(\boldsymbol{M}, \boldsymbol{t})}{\partial \boldsymbol{t}}=0 \rightarrow \boldsymbol{t}=\frac{1}{N} \sum_{i=1}^{N}\left[\boldsymbol{v}_{i}^{\mathrm{T}}\left(\boldsymbol{M} \boldsymbol{s}_{i}+\boldsymbol{t}\right) \boldsymbol{v}_{i}-\boldsymbol{M} \boldsymbol{s}_{i}\right]
$$

由 (9) 式可知

$$
\left(N \boldsymbol{I}-\sum_{i=1}^{N}\left(\boldsymbol{v}_{i} \boldsymbol{v}_{i}^{\mathrm{T}}\right)\right) \boldsymbol{t}=\sum_{i=1}^{N}\left(\boldsymbol{v}_{i} \boldsymbol{v}_{i}^{\mathrm{T}}-\boldsymbol{I}\right) \boldsymbol{M} \boldsymbol{s}_{i}
$$

其中 $\boldsymbol{I}$ 为 3 阶单位方阵.

可以证明 $N \boldsymbol{I}-\sum_{i=1}^{N}\left(\boldsymbol{v}_{i} \boldsymbol{v}_{i}^{\mathrm{T}}\right)$ 是一个正定矩阵.

证明 对于任意 3 维矢量 $\boldsymbol{x} \in \mathbb{R}^{3}$, 有

$$
\boldsymbol{x}^{\mathrm{T}}\left(N \boldsymbol{I}-\sum_{i=1}^{N}\left(\boldsymbol{v}_{i} \boldsymbol{v}_{i}^{\mathrm{T}}\right)\right) \boldsymbol{x}=\sum_{i=1}^{N}\left(\|\boldsymbol{x}\|^{2}-\boldsymbol{x}^{\mathrm{T}}\left(\boldsymbol{v}_{i} \boldsymbol{v}_{i}^{t}\right) \boldsymbol{x}\right)=\sum_{i=1}^{N}\left(\|\boldsymbol{x}\|^{2}-\left\|\boldsymbol{v}_{i}^{\mathrm{T}} \boldsymbol{x}\right\|^{2}\right) .
$$

$\boldsymbol{v}_{i}^{\mathrm{T}} \boldsymbol{x}$ 为矢量 $\boldsymbol{x}$ 在逆投影线 $\boldsymbol{v}_{i}$ 上的投影, 因此 $\|\boldsymbol{x}\|^{2}-\left\|\boldsymbol{v}_{i}^{\mathrm{T}} \boldsymbol{x}\right\|^{2}$ 都应大于或等于零, 当且仅当矢量 $\boldsymbol{x}$ 与所有的逆投影线 $\boldsymbol{v}_{i}$ 都平行, $\|\boldsymbol{x}\|^{2}-\left\|\boldsymbol{v}_{i}^{\mathrm{T}} \boldsymbol{x}\right\|^{2}$ 才可能都为零, 因此 $\|\boldsymbol{x}\|^{2}-\left\|\boldsymbol{v}_{i}^{\mathrm{T}} \boldsymbol{x}\right\|^{2}>0$, 进而可证明 $N \boldsymbol{I}-\sum_{i=1}^{N}\left(\boldsymbol{v}_{i} \boldsymbol{v}_{i}^{\mathrm{T}}\right)$ 是一个正定矩阵.

因 $N \boldsymbol{I}-\sum_{i=1}^{N}\left(\boldsymbol{v}_{i} \boldsymbol{v}_{i}^{\mathrm{T}}\right)$ 是正定矩阵, 其逆存在, 由 (10) 式得由旋转矩阵 $\boldsymbol{M}$ 表示的最优平移矢量 $\boldsymbol{t}(\boldsymbol{M})$ 为

$$
\boldsymbol{t}(\boldsymbol{M})=\left(N \boldsymbol{I}-\sum_{i=1}^{N}\left(\boldsymbol{v}_{i} \boldsymbol{v}_{i}^{\mathrm{T}}\right)\right)^{-1} \sum_{i=1}^{N}\left(\boldsymbol{v}_{i} \boldsymbol{v}_{i}^{\mathrm{T}}-\boldsymbol{I}\right) \boldsymbol{M} \boldsymbol{s}_{i} .
$$

在给出由旋转矩阵 $\boldsymbol{M}$ 表示的最优平移矢量 $\boldsymbol{t}(\boldsymbol{M})$ 后, 由 (4) 和 (5) 式可知各特征点的景深值也 可表示为旋转矩阵 $M$ 的函数:

$$
d_{i}(\boldsymbol{M})=\boldsymbol{v}_{i}^{\mathrm{T}}\left(\boldsymbol{M} \boldsymbol{s}_{i}+\boldsymbol{t}(\boldsymbol{M})\right) .
$$

同 (7) 式给出的景深估计算法相比, 本文所提出的算法利用了由旋转矩阵 $M$ 表示的最优平移矢 量 $\boldsymbol{t}(\boldsymbol{M})$, 能显著改善算法收敛性, 简单分析如下.

Haralick 算法中直接采用绝对方位解算给出的平移矢量求解各特征点景深值, 以本文采用的 Umeyama 算法为例分析直接采用该平移矢量的局限性. Umeyama 算法给出平移矢量求解公式如下 ${ }^{[18]}$ :

$$
\boldsymbol{t}=\boldsymbol{\mu}_{\boldsymbol{S}_{i}}^{\prime}-c \boldsymbol{M} \boldsymbol{\mu}_{\boldsymbol{s}_{i}}
$$

其中 $c=\frac{1}{\sigma_{s_{i}}^{2}} \operatorname{tr}(\boldsymbol{W} \boldsymbol{S}) ; \boldsymbol{\mu}_{\boldsymbol{S}_{i}^{\prime}}$ 可看作重构的各特征点重心坐标, 由 (4) 式知可表示为

$$
\mu_{S_{i}^{\prime}}=d \bar{v}
$$

$\boldsymbol{\mu}_{\boldsymbol{s}_{i}}$ 为已知的模型特征点重心坐标, 令 $\bar{s}=\boldsymbol{\mu}_{\boldsymbol{s}_{i}}$.

由 (14) 式可以看出, 在绝对方位解算中平移矢量是在转移矩阵确定之后计算出的, 因此可以将平 移矢量计算与绝对方位解算分离单独求解.

将 (15) 式代入 (14) 式得

$$
\boldsymbol{t}=d \overline{\boldsymbol{v}}-c \boldsymbol{M} \overline{\boldsymbol{s}}
$$

对比 (12) 与 (16) 式可知在满足如下条件时, (16) 式近似等于 (12) 式:

$$
c \approx 1,
$$




$$
\begin{aligned}
& \left(N \boldsymbol{I}-\sum_{i=1}^{N}\left(\boldsymbol{v}_{i} \boldsymbol{v}_{i}^{\mathrm{T}}\right)\right)^{-1} \approx \frac{\boldsymbol{I}}{N} \\
& \sum_{i=1}^{N}\left(\boldsymbol{v}_{i} \boldsymbol{v}_{i}^{\mathrm{T}}\right) \boldsymbol{M} \overline{\boldsymbol{v}} \approx N d \overline{\boldsymbol{v}}, d>0 .
\end{aligned}
$$

在特征点像点误差较小时, (17) 式成立. 若 (18) 式成立则要求特征点尽可能靠近光轴, 若 (19) 式 成立则要求所有特征点均位于与像平面平行的平面内, 即 (18)与 (19) 式成立的条件同能够利用弱透 视投影模型有效近似小孔成像模型的条件相一致, 因此 (16) 式是 (12) 式在弱透视投影假设下的近似. 然而, 在实际应用过程中, 采用弱透视投影假设会带来较大偏差, 因此利用 (12) 式代替 (14) 式能够有 效改善两阶段迭代算法的性能.

\section{1 .2 绝对方位解算}

计算出最优平移矢量 $\boldsymbol{t}(\boldsymbol{M})$ 并更新各特征点景深值 $d_{i}(\boldsymbol{M})$ 后, 可利用特征点景深重构特征点 $\boldsymbol{S}_{i}^{\prime}$ :

$$
\boldsymbol{S}_{i}^{\prime}(\boldsymbol{M})=d_{i}(\boldsymbol{M}) \boldsymbol{v}_{i}=\boldsymbol{v}_{i}^{\mathrm{T}}\left(\boldsymbol{M} \boldsymbol{s}_{i}+\boldsymbol{t}(\boldsymbol{M})\right) \boldsymbol{v}_{i}
$$

进而, (8) 式可以表示为

$$
e^{2}(\boldsymbol{M})=\sum_{i=1}^{N}\left\|\left(\boldsymbol{M} \boldsymbol{s}_{i}+\boldsymbol{t}(\boldsymbol{M})\right)-\boldsymbol{S}_{i}^{\prime}(\boldsymbol{M})\right\|^{2},
$$

其中旋转矩阵 $M$ 满足约束 $M M^{\mathrm{T}}=\boldsymbol{I}$.

(21) 式表示的受约束优化问题可看做是一个绝对方位问题. 绝对方位解算是摄影测量、计算机视 觉等领域的一个基础性问题, 要解决的是通过一定的特征点求解两个 $3 \mathrm{D}$ 模型所在坐标系间的平移和 旋转参数. 绝对方位解算方法可追溯到上世纪 60 年代摄影测量相关问题的求解, 特别是自上世纪 80 年代后出现了多种解析算法, Faugeras 和 Horn 分别提出的基于四元数的解析算法 ${ }^{[19,20]}$, Arun 等 [21] 提出的基于旋转矩阵奇异值分解的解析算法, 和 Umeyama ${ }^{[18]}$ 在 Arun 算法基础上的修正解析算法等.

由于 Umeyama 所提出的基于奇异值分解的修正算法在具有测量误差时的鲁棒性, 以及运算量小 等优点, 本文选择该算法进行绝对方位解算, 具体的 Umeyama 算法详见参考文献 [18].

\subsection{3 两阶段迭代算法流程}

综上, 基于特征点的空间目标三维位姿可通过如下迭代算法进行求解: 首先假设旋转矩阵第 $k$ 次 迭代值为 $\boldsymbol{M}^{(k)}$, 其次由 (12) 式计算最优平移矢量的第 $k$ 次迭代值 $\boldsymbol{t}^{\boldsymbol{M}(k)}=\boldsymbol{t}\left(\boldsymbol{M}^{(k)}\right)$, 再次由 (13) 式更新各特征点景深值 $d_{i}^{(k)}=d_{i}\left(\boldsymbol{M}^{(k)}, \boldsymbol{t}^{(k)}\right)$, 并由 (20) 式重构各特征点 $\boldsymbol{S}_{i}^{\prime(k)}=\boldsymbol{S}_{i}^{\prime}\left(d_{i}^{(k)}\right)$, 最后利用 Umeyama 解析算法求解旋转矩阵第 $k+1$ 次迭代值 $\boldsymbol{M}^{(k+1)}$, 即有

$$
\boldsymbol{M}^{(k+1)}=\arg \min _{(\boldsymbol{M})} \sum_{i=1}^{N}\left\|\left(\boldsymbol{M} \boldsymbol{s}_{i}+\boldsymbol{t}(\boldsymbol{M})\right)-\boldsymbol{S}_{i}^{\prime}(\boldsymbol{M})\right\|^{2} .
$$

两阶段迭代算法具体步骤如下:

(1) 初始化旋转矩阵 $\boldsymbol{M}^{(k)},(k=1)$, 由 (4) 式计算逆投影线单位矢量 $\boldsymbol{v}_{i}, i=1,2, \ldots, N$;

(2) 计算最优平移矢量

$$
\boldsymbol{t}^{(k)}=\left(N \boldsymbol{I}-\sum_{i=1}^{N}\left(\boldsymbol{v}_{i} \boldsymbol{v}_{i}^{\mathrm{T}}\right)\right)^{-1} \sum_{i=1}^{N}\left(\boldsymbol{v}_{i} \boldsymbol{v}_{i}^{\mathrm{T}}-\boldsymbol{I}\right) \boldsymbol{M}^{(k)} s_{i} ;
$$




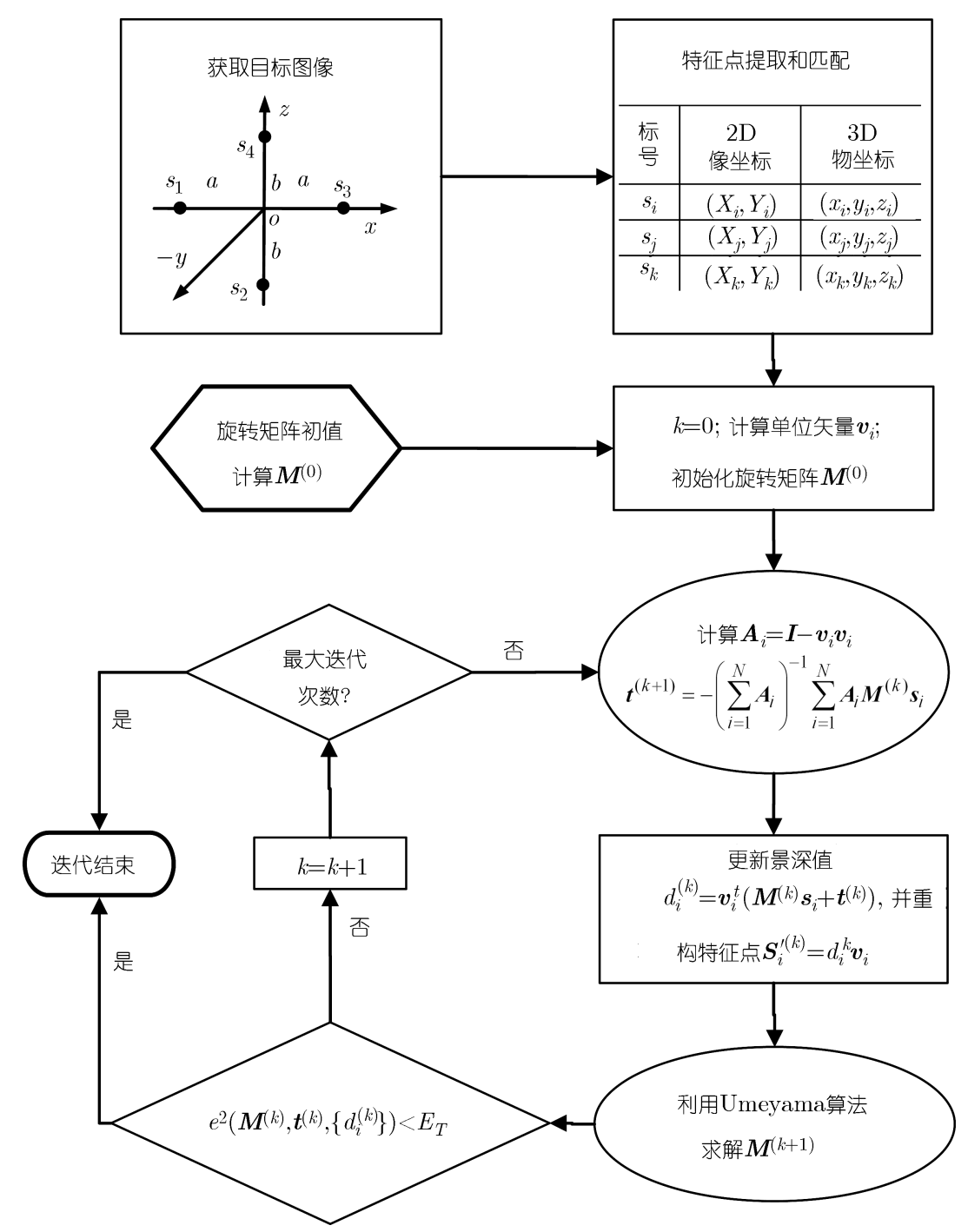

图 2 两阶段迭代算法流程图

(3) 更新景深值 $d_{i}^{(k)}=\boldsymbol{v}_{i}^{\mathrm{T}}\left(\boldsymbol{M}^{(k)} s_{i}+\boldsymbol{t}^{(k)}\right)$, 重构特征点 $\boldsymbol{S}_{i}{ }^{(k)}=d_{i}^{(k)} \boldsymbol{v}_{i}$;

(4) 采用 Umeyama 解析算法求解 (22) 式给出的绝对方位解算问题, 给出旋转矩阵 $\boldsymbol{M}^{(k+1)}$;

(5) 计算误差

$$
e^{2}\left(\boldsymbol{M}^{(k)}, \boldsymbol{t}^{(k)},\left\{d_{i}^{(k)}\right\}\right)=\sum_{i=1}^{N}\left\|d_{i}^{(k)} \boldsymbol{v}_{i}-\left(\boldsymbol{M}^{(k)} \boldsymbol{s}_{i}+\boldsymbol{t}^{(k)}\right)\right\|^{2} ;
$$

(6) 如果确定误差小于预定的误差阈值 $E_{T}$, 即 $e^{2}\left(\boldsymbol{M}^{(k)}, \boldsymbol{t}^{(k)},\left\{d_{i}^{(k)}\right\}\right)<E_{T}$, 则进入第 (8) 步, 否则, 置 $k:=k+1$;

(7) 若迭代次数少于最大迭代数, 返回到第 (2) 步, 否则进入第 (8) 步;

(8) 迭代结束, 输出结果.

算法流程图如图 2 所示, 从图中可以看出由于景深估计和绝对方位解算两阶段都是解析求解, 避 免了繁琐的非线性优化计算过程, 极大地降低了计算复杂度, 并具有良好的收玫性. 在算法推导过程 
中并没有利用任何特征光标点构型信息, 因此能够适用于 3 个以上不同特征点数、各种非共线构型测 量光标的空间目标三维位姿视觉测量任务.

\section{2 全局收敛性分析}

本节将利用全局收玫性定理证明所提出的两阶段迭代算法在任意给定一组目标特征像点和任意 初始值的情况下都能够收玫, 即该算法具有全局收玫性, 首先给出如下定义和全局收玫性定理 ${ }^{[22]}$.

定义 3.1 设 $X$ 和 $Y$ 分别是空间 $E_{p}$ 和 $E_{q}$ 中的非空闭集, $A: X \rightarrow Y$ 是点到集映射. 如果 (1) $x^{(k)} \rightarrow x, x^{(k)} \in X,(2) y^{(k)} \rightarrow y, y^{(k)} \in A\left(x^{(k)}\right)$. 蕴含 $y \in A(x)$, 则称映射 $A$ 在 $x \in X$ 处是闭的. 如果 映射 $A$ 在集合 $Z \subset X$ 内每一点都是闭的, 则称映射 $A$ 在集合 $Z$ 内是闭的.

定理 3.1 设 $A$ 为 $X$ 上的一个算法, $\Omega$ 为解集合, 给定初值 $x^{(0)} \in X$, 进行如下迭代: 若 $x^{(k)} \in \Omega$, 则停止迭代; 否则, 令 $x^{(k+1)} \in A\left(x^{(k)}\right)$. 用 $k+1$ 代替 $k$, 重复以上过程, 产生序列 $\left\{x^{(k)}\right\}$. 又设 (1) 映 射 $A$ 在 $\Omega$ 的补集上是闭的; (2) 序列 $\left\{x^{(k)}\right\}$ 含于 $X$ 的紧子集中; (3) 存在一个连续函数, 它是关于 $\Omega$ 和 $A$ 的下降函数. 则序列 $\left\{x^{(k)}\right\}$ 的任一收玫子序列的极限属于 $\Omega$.

本文提出的两阶段迭代算法可以看作是由第 $k$ 次迭代值 $\boldsymbol{M}^{(k)}$ 到第 $k+1$ 次迭代值 $\boldsymbol{M}^{(k+1)}$ 的映 射, 其中 $\boldsymbol{M}^{(k)} \in \mathcal{S O}(3)$, 根据定理 3.1, 为证明本文所提出的两阶段迭代算法具有全局收玫性, 需要证 明该算法满足定理中的 3 条假设.

证明 因算法中步骤 (2) 和 (3) 是连续的点到点映射, 故其是一闭点到集映射, 且步骤 (4) 中的 绝对方位确定算法也已被证明是一闭映射 ${ }^{[15]}$, 因此两阶段迭代算法全局收玫的第一个条件是满足的.

现证其满足第 2 个条件, 注意到两阶段迭代算法的输出本质上是 $\boldsymbol{M}^{(k+1)}$, 由 SVD 分解性质可知: $\boldsymbol{U}$ 和 $\boldsymbol{V}$ 为正交矩阵. 而注意到 $\boldsymbol{S}=\boldsymbol{S}^{\prime}$, 且有 $\boldsymbol{S} \boldsymbol{S}^{\prime}=\boldsymbol{I}$, 所以 $\boldsymbol{M}^{(k+1)}\left(\boldsymbol{M}^{(k+1)}\right)^{\mathrm{T}}=\boldsymbol{I}$, 故 $\boldsymbol{M}^{(k+1)}$ 为正 交阵. 由于正交矩阵是紧集 (闭且有界), 因此第 2 个条件也满足.

下面证明第 3 个条件也是满足的, 迭代算法第 $k+1$ 次迭代后的目标函数误差可由第 $k$ 次迭代后 位姿参数表示为

$$
\begin{aligned}
e_{k+1}^{2}( & \left.\boldsymbol{M}^{(k+1)}, \boldsymbol{t}^{(k+1)},\left\{d_{i}^{(k+1)}\right\}\right)=\sum_{i=1}^{N}\left\|\boldsymbol{M}^{(k+1)} \boldsymbol{s}_{i}+\boldsymbol{t}^{(k+1)}-d_{i}^{(k+1)} \boldsymbol{v}_{i}\right\|^{2} \\
= & \sum_{i=1}^{N}\left\|\boldsymbol{M}^{(k+1)} \boldsymbol{s}_{i}+\boldsymbol{t}^{(k+1)}-d_{i}^{(k)} \boldsymbol{v}_{i}\right\|^{2}+\sum_{i=1}^{N}\left(d_{i}^{(k)}-d_{i}^{(k+1)}\right)\left[2 \boldsymbol { v } _ { i } ^ { t } \left(\boldsymbol{M}^{(k+1)} \boldsymbol{s}_{i}\right.\right. \\
& \left.\left.+\boldsymbol{t}^{(k+1)}-d_{i}^{(k)} \boldsymbol{v}_{i}\right)+\left(d_{i}^{(k)}-d_{i}^{(k+1)}\right)\left\|\boldsymbol{v}_{i}\right\|^{2}\right] .
\end{aligned}
$$

由于 $\boldsymbol{v}_{i}$ 为逆投影线方向单位矢量, 因此有 $\left\|\boldsymbol{v}_{i}\right\|^{2}=1$, 进而 (23) 式等式右端第 2 项可表示为

$$
\begin{aligned}
& \sum_{i=1}^{N}\left(d_{i}^{(k)}-d_{i}^{(k+1)}\right)\left[2 \boldsymbol{v}_{i}^{t}\left(\boldsymbol{M}^{(k+1)} \boldsymbol{s}_{i}+\boldsymbol{t}^{(k+1)}\right)-d_{i}^{(k)}-d_{i}^{(k+1)}\right] \\
& \quad=\sum_{i=1}^{N}\left\{\left(d_{i}^{(k+1)}\right)^{2}-2 d_{i}^{(k+1)} \boldsymbol{v}_{i}^{t}\left(\boldsymbol{M}^{(k+1)} \boldsymbol{s}_{i}+\boldsymbol{t}^{(k+1)}\right)+2 d_{i}^{(k)} \boldsymbol{v}_{i}^{t}\left(\boldsymbol{M}^{(k+1)} \boldsymbol{s}_{i}+\boldsymbol{t}^{(k+1)}\right)-\left(d_{i}^{(k)}\right)^{2}\right\} \\
& \quad=\sum_{i=1}^{N}\left\{\left\|d_{i}^{(k+1)}-\boldsymbol{v}_{i}^{t}\left(\boldsymbol{M}^{(k+1)} \boldsymbol{s}_{i}+\boldsymbol{t}^{(k+1)}\right)\right\|^{2}-\left\|d_{i}^{(k)}-\boldsymbol{v}_{i}^{t}\left(\boldsymbol{M}^{(k+1)} \boldsymbol{s}_{i}+\boldsymbol{t}^{(k+1)}\right)\right\|^{2}\right\} .
\end{aligned}
$$

由于利用 Umeyama 算法给出的 $\boldsymbol{M}^{(k+1)}$ 是最小二乘意义下的最优解, 使 (21) 式表示的目标函数 
取最小值, 即有

$$
\boldsymbol{M}^{(k+1)}=\arg \min _{(\boldsymbol{M})} \sum_{i=1}^{N}\left\|\left(\boldsymbol{M} \boldsymbol{s}_{i}+\boldsymbol{t}(\boldsymbol{M})\right)-d_{i}\left(\boldsymbol{M}^{(k)}\right) \boldsymbol{v}_{i}\right\|^{2}
$$

因此有

$$
\sum_{i=1}^{N}\left\|\boldsymbol{M}^{(k+1)} \boldsymbol{s}_{i}+\boldsymbol{t}^{(k+1)}-d_{i}^{(k)} \boldsymbol{v}_{i}\right\|^{2} \leqslant \sum_{i=1}^{N}\left\|\boldsymbol{M}^{(k)} \boldsymbol{s}_{i}+\boldsymbol{t}^{(k)}-d_{i}^{(k)} \boldsymbol{v}_{i}\right\|^{2}=e_{k}^{2}\left(\boldsymbol{M}^{(k)}, \boldsymbol{t}^{(k)},\left\{d_{i}^{(k)}\right\}\right) .
$$

将 (13) 式代入 (24) 式, 进而连同 (26) 式一起代入 (23) 式可得

$$
e_{k+1}^{2}\left(\boldsymbol{M}^{(k+1)}, \boldsymbol{t}^{(k+1)},\left\{d_{i}^{(k+1)}\right\}\right) \leqslant e_{k}^{2}\left(\boldsymbol{M}^{(k)}, \boldsymbol{t}^{(k)},\left\{d_{i}^{(k)}\right\}\right)-\sum_{i=1}^{N}\left[d_{i}^{(k)}-\boldsymbol{v}_{i}^{t}\left(\boldsymbol{M}^{(k+1)} \boldsymbol{s}_{i}+\boldsymbol{t}^{(k+1)}\right)\right]^{2} .
$$

(27) 式中不等式右端第 2 项不可能为负, 且 (26) 式中当且仅当 $\boldsymbol{M}^{(k)}, \boldsymbol{t}^{(k)}$ 和 $\boldsymbol{M}^{(k+1)}, \boldsymbol{t}^{(k+1)}$ 完全 相同时才取等号, 因此在算法迭代过程中有

$$
e_{k+1}^{2}\left(\boldsymbol{M}^{(k+1)}, \boldsymbol{t}^{(k+1)},\left\{d_{i}^{(k+1)}\right\}\right)<e_{k}^{2}\left(\boldsymbol{M}^{(k)}, \boldsymbol{t}^{(k)},\left\{d_{i}^{(k)}\right\}\right) .
$$

(28) 式表明算法在收玫到稳定点之前, 目标函数是严格递减的, 因此迭代算法全局收玫的第 3 个 条件也能够满足, 进而根据全局收玫性定理可以证明本文算法具有全局收敛性.

\section{4 仿真与结果分析}

航天器间相对位置、相对姿态等运动状态的精确测量是实现航天器交会对接的关键技术之一, 单 目视觉测量系统是在跟踪航天器上安装视觉相机, 在目标航天器上安装几何形状及尺寸已知的特征光 标, 通过对特征光标点的分析和计算精确确定追踪航天器和目标航天器间的相对位置和姿态等参数, 在空间交会对接的最后逼近段及停靠对接段得到了广泛应用 ${ }^{[1]}$.

为验证本文所提出的两阶段迭代算法的有效性, 以航天器交会对接中的视觉测量任务为背景, 对 其进行数值和物理仿真.

\section{1 数学仿真}

目标航天器上特征光标 $s_{i}:\left(x_{i}, y_{i}, z_{i}\right)^{\mathrm{T}}, i=1, \ldots, 4$ 在目标坐标系中的坐标分别为

$$
\begin{aligned}
& s_{1}:\left[\begin{array}{l}
x_{1} \\
y_{1} \\
z_{1}
\end{array}\right]=\left[\begin{array}{c}
0 \\
-0.2 \\
0
\end{array}\right] m, s_{2}:\left[\begin{array}{l}
x_{2} \\
y_{2} \\
z_{2}
\end{array}\right]=\left[\begin{array}{c}
-0.2 \\
0 \\
0
\end{array}\right] m, \\
& s_{3}:\left[\begin{array}{l}
x_{3} \\
y_{3} \\
z_{3}
\end{array}\right]=\left[\begin{array}{c}
0.2 \\
0 \\
0
\end{array}\right] m, s_{4}:\left[\begin{array}{l}
x_{4} \\
y_{4} \\
z_{4}
\end{array}\right]=\left[\begin{array}{c}
0 \\
0.2 \\
0
\end{array}\right] m .
\end{aligned}
$$

假定两对接航天器相对姿态为 $[\phi, \theta, \psi]=\left[10^{\circ}, 15^{\circ}, 8^{\circ}\right]$, 相对位置为 $\boldsymbol{t}=[1 \mathrm{~m},-0.05 \mathrm{~m}, 0.45 \mathrm{~m}]^{\mathrm{T}}$, 特 征点提取误差为 2 像素. 在上述初始条件下, 分别对本文给出的两阶段迭代算法以及文献 [14] 给出的 

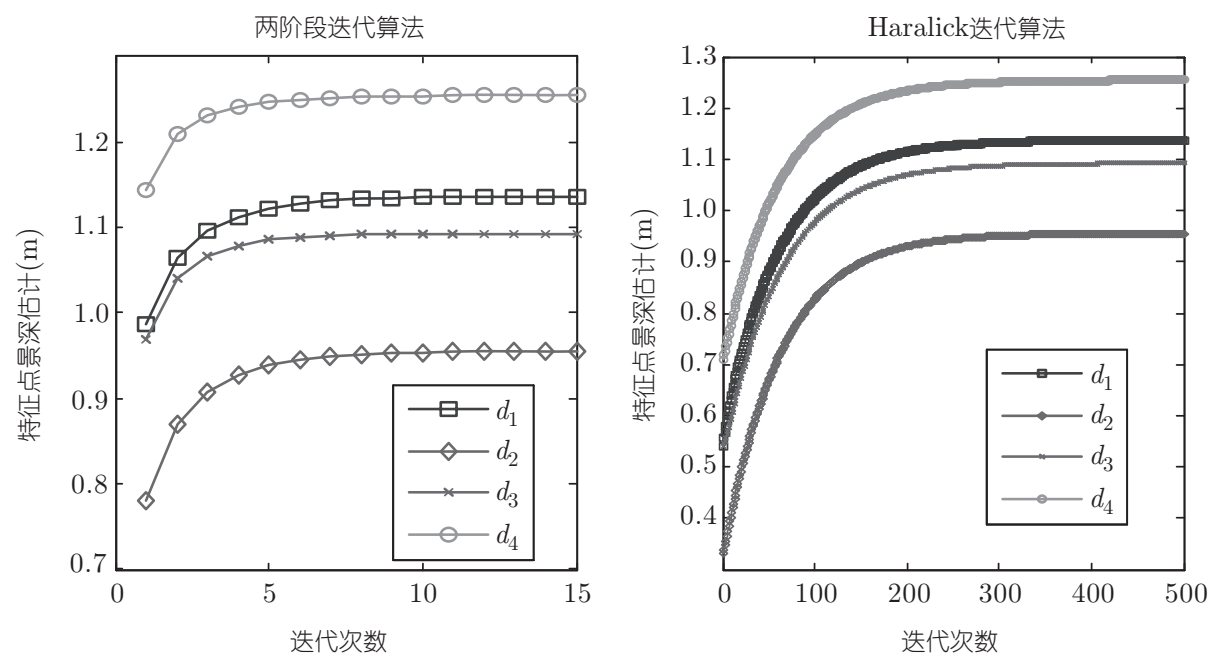

图 3 单次迭代过程中各特征点景深变化曲线
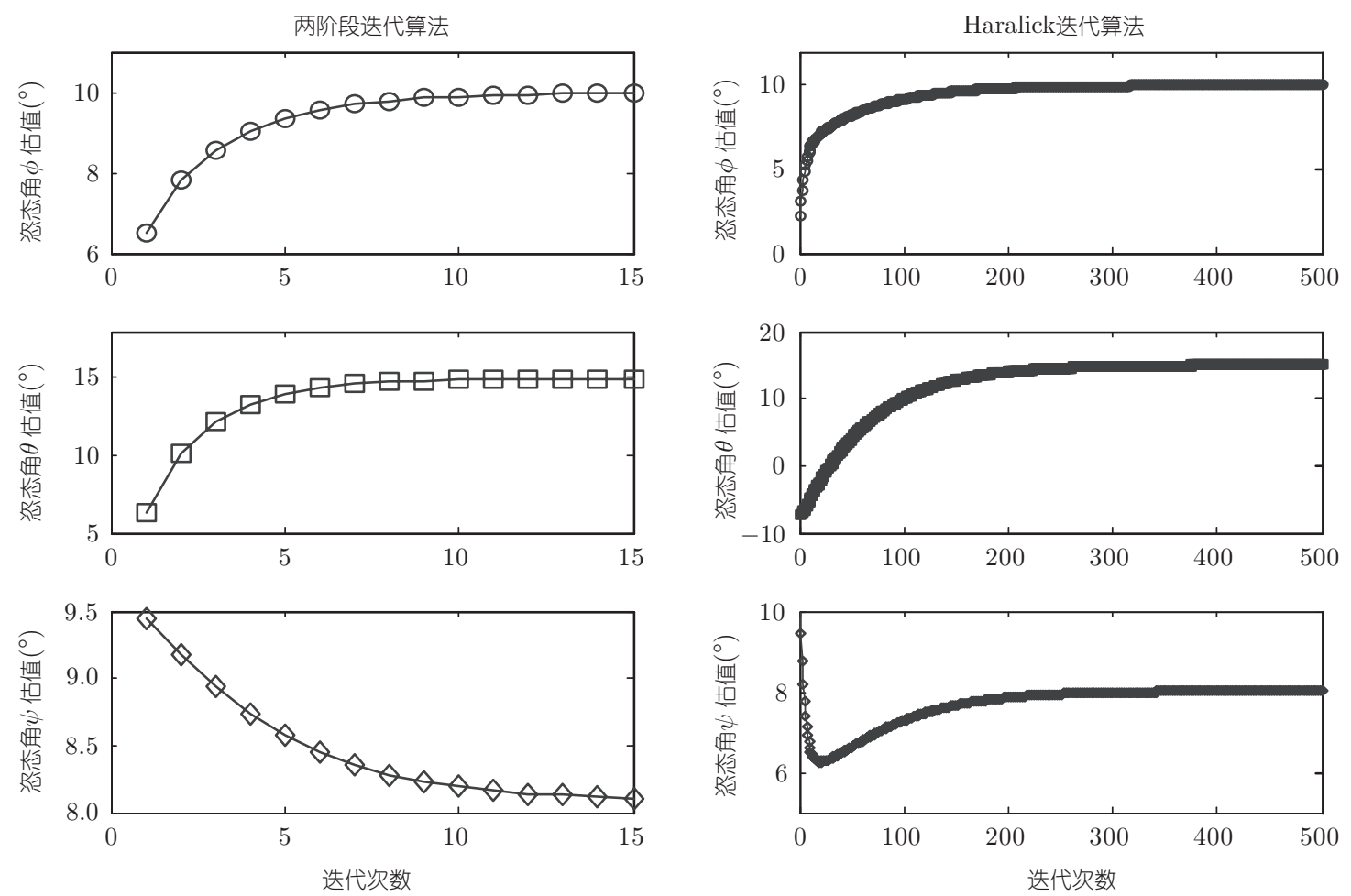

图 4 单次迭代过程中相对姿态变化曲线

Haralick 迭代算法进行仿真. 两阶段迭代算法旋转矩阵迭代初值 $\boldsymbol{M}^{(0)}=\boldsymbol{I}_{3 \times 3}$, Haralick 迭代算法的 特征点景深迭代初值 $d_{1}^{(1)}=0.54, d_{2}^{(1)}=0.33, d_{3}^{(1)}=0.54, d_{4}^{(1)}=0.7$, 两算法结束的误差阈值均设为 $1 \times 10^{-7}$, 算法运行结果如图 3 5 所示.

从图 3 5 可以看出在较大初值偏差情况下, 本文所提出的两阶段迭代算法在 15 次迭代之内能够 收玫到正确值, 远快于 Haralick 迭代算法的 500 余次迭代, 验证了该算法的全局收敛性和快速性. 

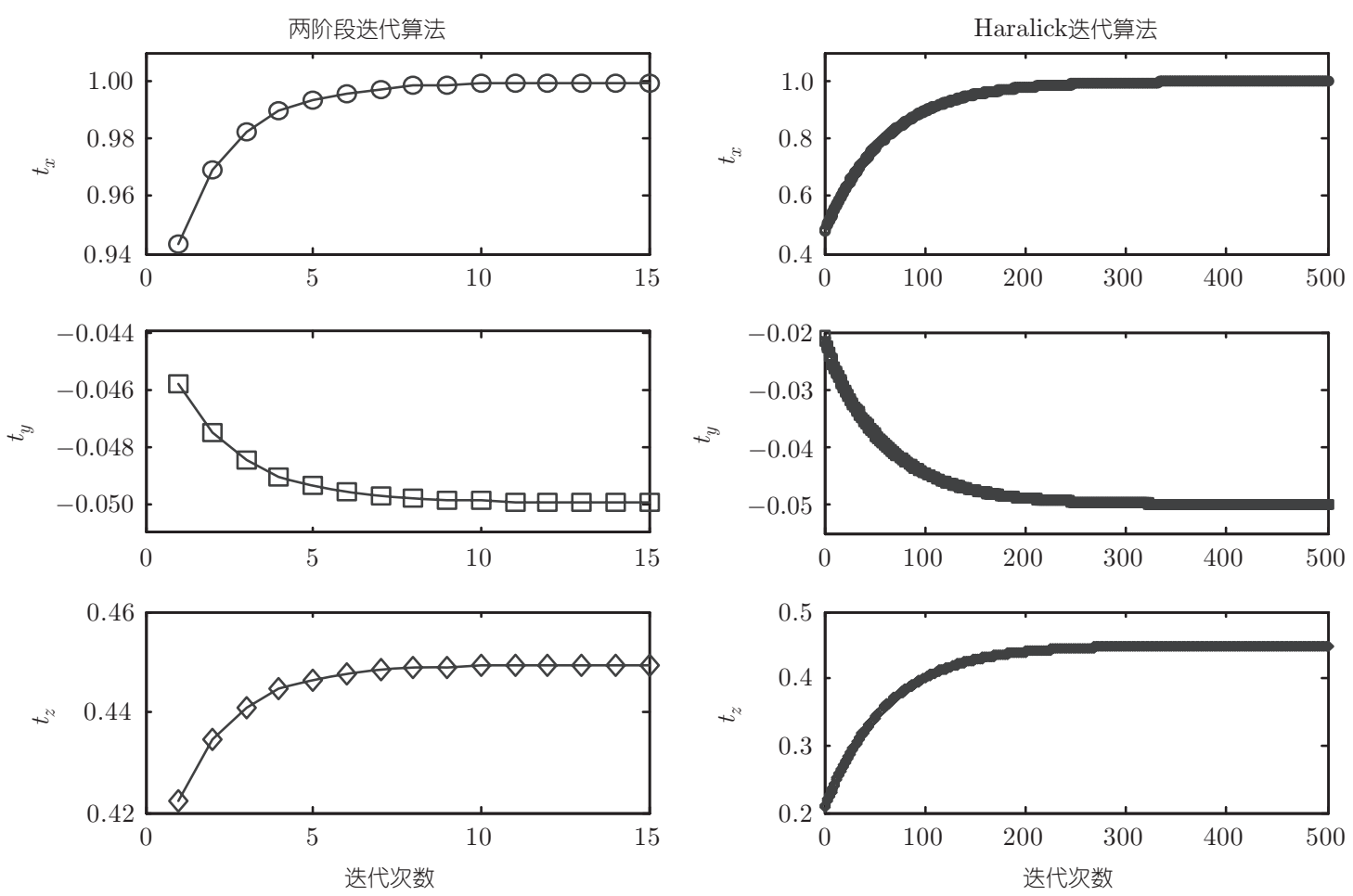

图 5 单次迭代过程中相对位置变化曲线

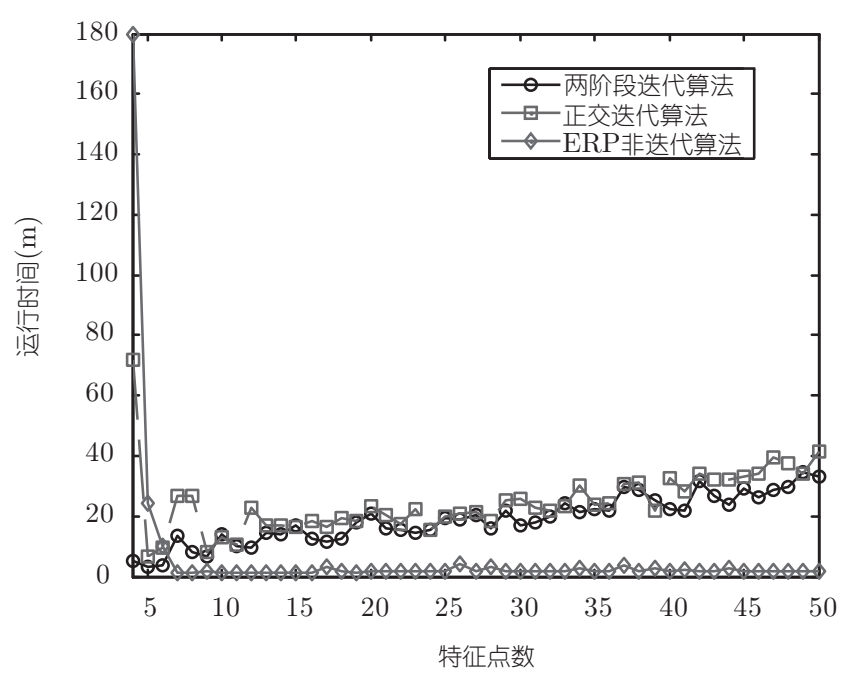

图 6 运行时间与特征点数关系

第 2 例仿真验证考虑算法的计算效率, Lu 等提出的正交迭代 (orthogonal iteration) 算法和 Lepetit 等提出的 $\mathrm{EP} n \mathrm{P}$ 算法分别是是目前运行速度快、结果精确的迭代算法与非迭代算法之一 ${ }^{[11,15]}$, 将上 述两算法同两阶段迭代算法作比较. 运行软件为 Matlab ${ }^{\circledR} 6.5$, 硬件是 $2.2 \mathrm{GHz}$ 双核处理器、2 $\mathrm{GB}$ 内 存的台式机. 为估计运行时间, 针对各种特征点数均运行 100 次求平均值, 结果如图 6 所示.

从图 6 可看出两阶段迭代算法运行时间随参与特征点数的增多而近似线性增长, 且与正交迭代算 法运算速度相当, 稍慢于 $\mathrm{EP} n \mathrm{P}$ 非迭代算法. 然而, 在算法运行中两阶段迭代算法与正交迭代算法结 束的误差阈值均设为 $1 \times 10^{-7}$, 因此两算法的精度大致相当, $\mathrm{EP} n \mathrm{P}$ 算法的精度较上述两算法低很多. 


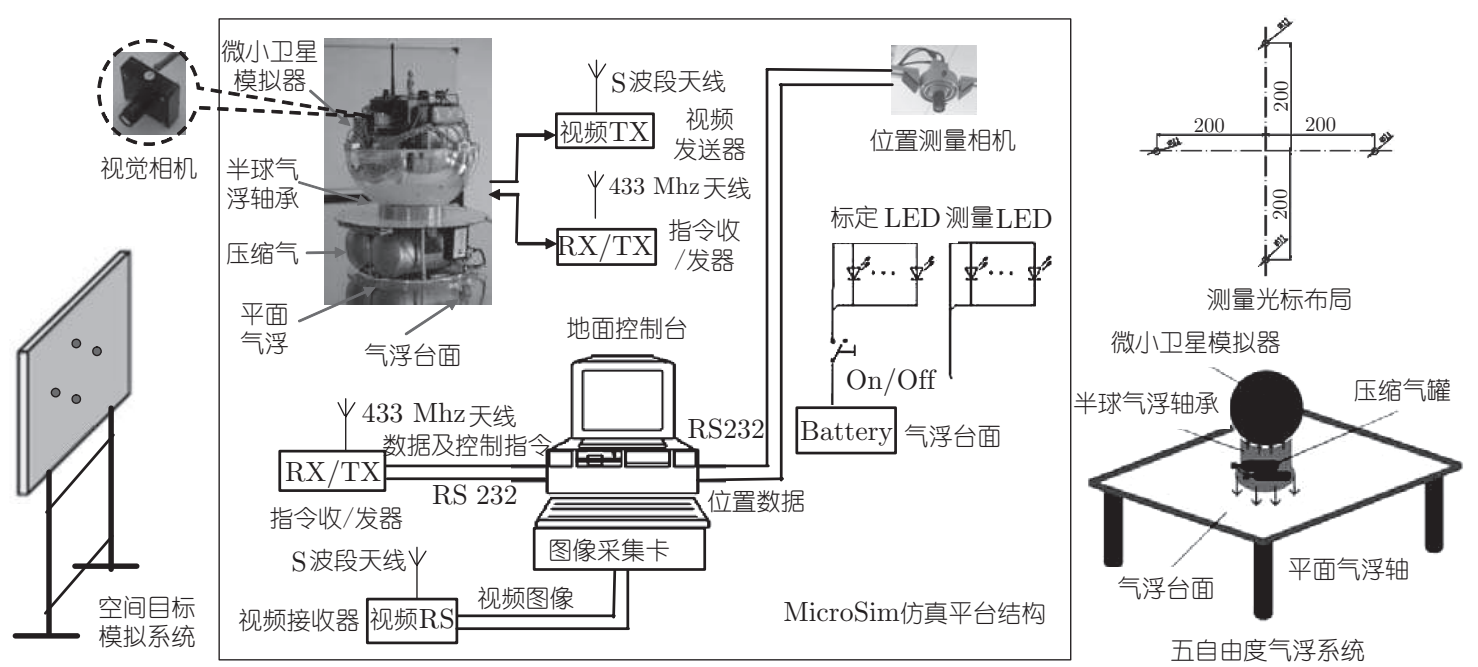

图 7 基于 MicroSim 的单目视觉测量物理仿真系统

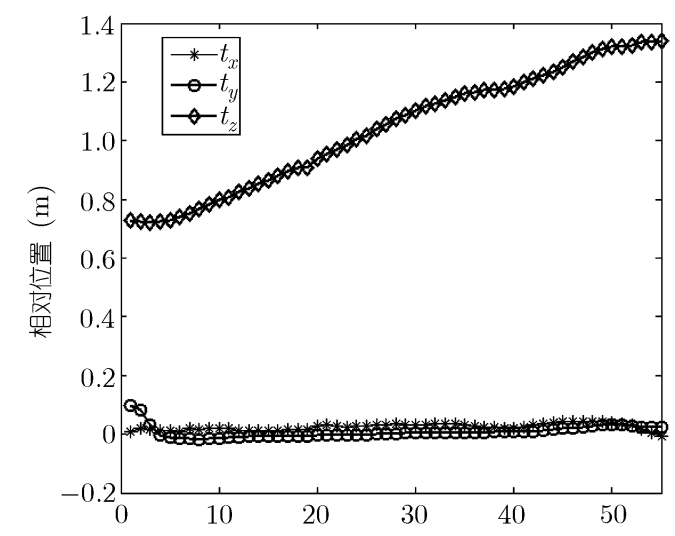

(a) 相对位置估计值

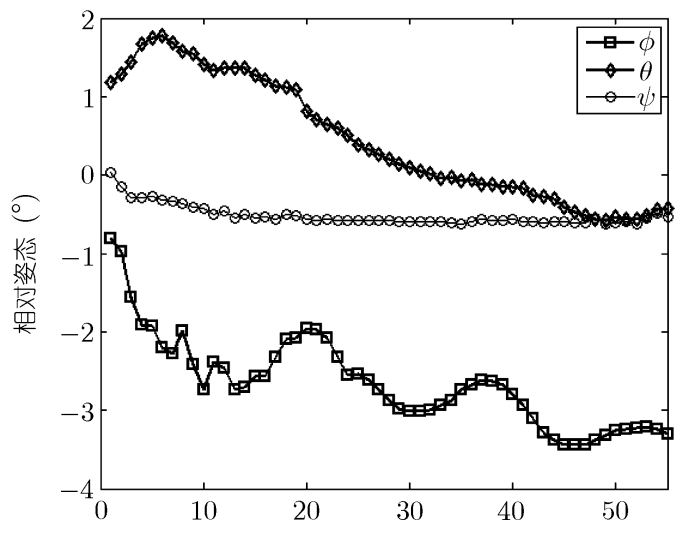

(b) 相对位置估计值

图 8 物理仿真相对位姿确定结果

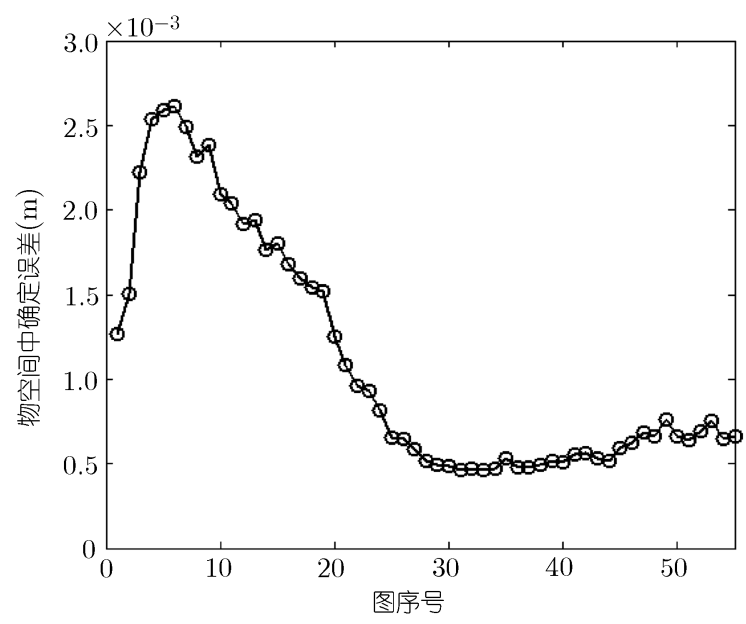

图 9 物空间中确定误差曲线 


\section{2 物理仿真}

MicroSim 仿真平台是基于气浮原理建立的卫星地面物理仿真环境, 由运动模拟系统、微小卫星系 统、地面测量系统及地面控制台等构成 ${ }^{[23]}$. 运动模拟系统具有三轴球形气浮轴承和平面气浮台, 可提 供三轴旋转旋转和两轴平移的无干扰环境, 实现卫星在轨姿态和轨道运动模拟. 微小卫星系统包含了 陀螺和加速度计等敏感器、反作用飞轮和冷喷气系统等执行机构、星载计算机及星地通信系统等真实 卫星的所有分系统, 还装有 3 个微视觉相机. MicroSim 仿真平台能够用于航天器交会对接相对导航与 控制等关键技术的地面物理仿真验证. 基于 MicroSim 仿真平台进行单目视觉测量地面物理仿真以进 一步验证本文算法的有效性, MicroSim 仿真平台及测量光标系统如图 7 所示.

控制微小卫星系统沿视觉相机 $Z$ 方向远离测量光标, 并等间隔保存 55 幅测量光标图像, 利用 Tsai 标定算法对视觉相机内部参数进行标定, 进而对所获取的 55 幅图像进行处理和特征点提取, 并输入 两阶段迭代算法以确定测量光标相对于视觉相机的相对位置和姿态, 结果如图 8 所示.

由 (6) 式表示的目标函数值的平方根可近似看作各特征点在物空间中确定误差的算术平方根, 能 在一定程度上表征确定算法的精度, 物理仿真中各特征点在物空间中的确定误差如图 9 所示.

从图 9 可以看出本文所提出的两阶段迭代算法的确定误差能够达到亚毫米量级, 通过物理仿真进 一步验证了本文算法的有效性和快速收敛性.

\section{5 结论}

受逆投影线思想的启发, 本文提出了一种基于特征点的两阶段迭代空间目标三维位姿单目视觉确 定算法, 该算法包含景深估计以及绝对方位解算两阶段, 上述两阶段迭代进行直至结果收玫. 同传统 的基于非线性优化算法的单目视觉相对位姿确定算法相比, 由于该算法是迭代进行的代数求解过程, 克服了传统算法所面临的计算量大、收玫性差等缺点. 通过理论证明了本文算法的全局收玫性, 并以 航天器交会对接中的单目视觉测量任务为应用背景, 通过数学和物理仿真进一步验证了本文算法的有 效性和快速收玫性. 由于本文算法推导过程中没有利用任何光标点的几何构型信息, 因此能够适应不 同配置特征光标的视觉测量任务.

致谢 作者对成都电子科技大学郝家胜博士在论文写作过程中提供的大力帮助深表感谢.

\section{参考文献}

1 Philip N K, Ananthasayanam M R. Relative position and attitude estimation and control schemes for the final phase of an autonomous docking mission of spacecraft. Acta Astronaut, 2003, 52: 511-522

2 Wu Y H, Hu Z Y. PnP problem revisited. J Math Imag Vis, 2006, 24: 131-141

3 Gao X S, Hou X R, Tang J, et al. Complete solution classification for the perspective-three-point problem. IEEE Trans Pattern Anal, 2003, 25: 930-943

$4 \mathrm{Hu}$ Z Y, Wu C F. A note on the number of solutions of the noncoplanar P4P problem. IEEE Trans Pattern Anal, 2002, 24: $550-555$

5 Nister D. An efficient solution to the five-point relative pose problem. IEEE Trans Pattern Anal, 2004, 26: 756-770

6 Quan L, Lan Z D. Linear N-point camera pose determination. IEEE Trans Pattern Anal, 1999, 21: 1-7

7 Tang J L, Chen W S, Wang J. A novel linear algorithm for P5P problem. Appl Math Comput, 2008, 205: 628-634

8 Ansar A, Daniilidis K. Linear pose estimation from points or lines. IEEE Trans Pattern Anal, 2003, 25: 578-589 
9 Kúkelová Z, Bujňák M, Pajdla T. Polynomial eigenvalue solutions to the 5-pt and 6-pt relative pose problems. In: BMVC 2008: Proceedings of the 19th British Machine Vision Conference. London: British Machine Vision Association, 2008. 565-574

10 Paul D F. Efficient linear solution of exterior orientation. IEEE Trans Pattern Anal, 2001, 23: 140-148

11 Lepetit V, Moreno-Noguer F, Fua P. EP $n \mathrm{P}$ : an accurate $\mathrm{O}(n)$ solution to the $\mathrm{P} n \mathrm{P}$ problem. Int J Comput Vision, 2009, 81: 155-166

12 Lee P Y, Moore J B. Gauss-Newton-on-manifold for pose estimation. J Ind Manage Optim, 2005, 1: 565-587

13 Dementhon D, Davis L S. Model-based object pose in 25 lines of code. Int J Comput Vision, 1995, 15: 123-141

14 Haralick R M, Joo H, Lee C N, et al. Pose estimation from corresponding point data. IEEE Trans Syst Man Cy, 1989, 19: $1426-1446$

15 Lu C P, Hager G D, Mjolsness E. Fast and globally convergent pose estimation from video images. IEEE Trans Pattern Anal, 2000, 22: 610-622

16 Schweighofer G, Pinz A. Robust pose estimation from a planar target. IEEE Trans Pattern Anal, 2006, 28: 2024-2030

17 Schweighofer G, Pinz A. Globally optimal $\mathrm{O}(n)$ solution to the P $n$ P problem for general camera models. In: Everingham M, Needham C J, eds. Proceedings of the 19th British Machine Vision Conference. London: BMVA Press, 2008. 1-10

18 Umeyama S. Least-squares estimation of transformation parameters between two point patterns. IEEE Trans Pattern Anal, 1991, 13: 367-380

19 Faugeras O D, Hebert M. The representation, recognition, and locating of 3D shapes from range data. Int J Robot Res, 1986, 5: 27-52

20 Horn B K P. Closed-form solution of absolute orientation using unit quaternion. J Opt Soc Am A, 1987, 4: 629-642

21 Arun K S, Huang T S, Blostein S D. Least-squares fitting of two 3-d point sets. IEEE Trans Pattern Anal, 1987, 9: 698-700

22 Luenberger D G, Ye Y Y. Linear and Nonlinear Programming. 3rd ed. New York: Springer, 2008. 201-208

23 张世杰, 曹喜滨. 基于 MicroSim 仿真平台的航天器交会对接物理仿真系统. 航天控制, 2006, 24: 63-67, 96 